\title{
Existence of Solutions to a Generalized System*
}

\author{
Yali Zhao, Lin Xing ${ }^{\#}$, Jia Tao \\ Department of Mathematics, Bohai University, Jinzhou, China \\ Email: yalizhao2000@yahoo.com.cn, ${ }^{\# x i n g l i n 251214 @ y e a h . n e t ~}$
}

Received February 19, 2012; revised April 10, 2012; accepted April 17, 2012

\begin{abstract}
In this paper, we introduce a generalized system (for short, GS) in real Banach spaces. Using Brouwer's fixed point theorem, we establish some existence theorems for the generalized system without monotonicity. Further, we extend the concept of C-strong pseudomonotonicity and extend Minty's lemma for the generalized system. And using the Minty lemma and KKM-Fan lemma, we establish an existence theorem for the generalized system with monotonicity in real reflexive Banach spaces. As the continuation of existing studies, our paper present a series of extended results based on existing corresponding results.
\end{abstract}

Keywords: Generalized System; C-Strong Pseudomonotonicity; Brouwer's Fixed Point Theorem; Hemicontinuous Mapping

\section{Introduction}

Variational inequality theory has played a fundamental and important role in the study of a wide range of problems arising in physics, mechanics, differential equations, contact problems in elasticity, optimization, economics and engineering sciences, etc. As a useful and important branch of variational inequality theory, vector variational inequalities were initially introduced and considered by Giannessi [1] in a finite-dimensional space in 1980. Ever since then, vector variational inequalities have been extensively studied and generalized in infinite-dimensional spaces.

Very recently, Fang and Huang [2] studied some existence results for strong vector variational inequalities in Banach spaces. Long, Huang and Teo [3] established an existence theorem of solutions for a generalized strong vector quasi-equilibrium problem by using KakutaniFan-Glicksberg fixed point theorem. $\mathrm{Li}$ and He [4] studied the existence of solutions for VVI with a single-valued function and a continuous selection theorem and obtained the existence theorem for the GVVI under the assumption of $\eta-h-C(x)$-pseudomonotonicity.

Motivated and inspired by research works mentioned above, in this paper, we consider a generalized system, which includes as special cases the strong vector variational inequalities $[1,2]$ and problems so called equilibrium problems $[5,6]$. In order to derive the existence of

\footnotetext{
${ }^{*}$ This work is supported by the Doctoral Initiating Foundation of Liaoning Province (20071097) and partially supported by the National Natural Science Foundation of China (61070242).

${ }^{\#}$ Corresponding author.
}

solutions for the generalized system, Using Brouwer's fixed point theorem, we obtain some existence results for the generalized system without monotonicity. Furthermore, by the concepts of - $C$-continuous, $C$-strong pseudomonotonicity and hemicontinuity, we extend Minty's lemma, moreover, with the help of Minty's lemma and KKM-Fan lemma, we establish an existence theorem for the generalized system with monotonicity in real reflexive Banach spaces. The results presented in this paper extend the corresponding results of $[2,6]$, and Theorems 3.1-3.3 can be considered as a generalization of Theorems 2.1-2.3 in [6].

\section{Preliminaries}

Throughout the paper, otherwise special statement, let $X$ and $Y$ be two real Banach spaces, let $K \subset X$ be a nonempty, closed and convex set, let $C \subset Y$ be a solid, pointed, closed and convex cone with apex at the origin. Let $T: K \rightarrow 2^{L(X, Y)}, L(X, Y)$ be the space of all continuous linear mappings from $X$ to $Y$. Let

$f: L(X, Y) \times K \times K \rightarrow Y$ be a trifunction such that $f(s, x, x)=0$ for all $x \in K$ and $s \in T x$. Then we consider the following generalized systems (for short, GS): Find $x \in K$, for each $y \in k$ there exists $s \in T x$ such that

$$
f(s, y, x) \notin-C \backslash\{0\} .
$$

And find $x \in K, s \in T x$ such that

$$
f(s, y, x) \notin-C \backslash\{0\}, \forall y \in K .
$$


We know that a solution $(x, s) \in K \times L(X, Y)$ is called a weak solution and a strong solution to GS(1) and GS(2), respectively. It is easy to see that a solution to $\mathrm{GS}(2)$ is a solution to $\mathrm{GS}(1)$, but in general the converse is not true. Noting $f(s, y, x)=\langle T x, \eta(y, x)\rangle, \forall x, y \in K$, where $T: K \rightarrow L(X, Y)$ is a single-valued mapping, $\eta: K \times K \rightarrow X$ is a nonlinear mapping, then GS(1)and GS(2) both reduces to the following strong vector variational-like inequality problem (for short, SVVLIP): Find $x \in K$ such that

$$
\langle T x, \eta(y, x)\rangle \notin-C \backslash\{0\}, \forall y \in K .
$$

Now, we recall the following definitions.

Definition 2.1 [7]. Let $K$ be a subset of a topological vector space $X$. A set-valued mapping $T: K \rightarrow 2^{X}$ is called a KKM mapping if, for each nonempty finite subset $\left\{x_{1}, x_{2}, \cdots, x_{n}\right\} \subset K$, we have

$$
\operatorname{Co}\left\{x_{1}, \cdots, x_{n}\right\} \subset \bigcup_{i=1}^{n} T\left(x_{i}\right),
$$

where $C o A$ denotes the convex hull of the set $A$.

Definition 2.2. Let $X$ and $Y$ be topological vector spaces, let $K$ be a nonempty, convex subset of $X$ and let $C$ be a convex cone in $Y$. A mapping $g: K \rightarrow Y$ is said to be $C$-convex function if, for all $\lambda \in[0,1]$ and $x_{1}$, $x_{2} \in K$,

$$
\lambda g\left(x_{1}\right)+(1-\lambda) g\left(x_{2}\right)-g\left(\lambda x_{1}+(1-\lambda) x_{2}\right) \in C .
$$

Remark 2.1. 1) If $C$ contains (or is equal to,or is contained in) the non-negative orthant, then the $C$-convex function is called $C$-convex (or convex, or strictly $C$ convex);

2) If $C$ contains (or is equal to, or is contained in) the nonpositive orthant, then the $C$-convex function is called $C$-concave (or concave, or strictly $C$-concave).

Theorem 2.1. (Brouwer's fixed point theorem [8]) Let $K$ be a nonempty, compact and convex subset of a finite-dimensional space $X$ and let $f: K \rightarrow K$ be a continuous mapping. Then, there exists $x \in K$ such that $f(x)=x$.

Theorem 2.2. (KKM-Fan Lemma [7]) Let $K$ be a subset of a topological vector space $X$ and let $T: K \rightarrow 2^{X}$ be a KKM mapping. If, for each $x \in K, T(x)$ is closed and for at least one $x \in K, T(x)$ is compact, then $\bigcap_{x \in K} T(x) \neq \varnothing$.

\section{Main Results}

In this section, we shall obtain the following existence theorems for GS(1).

Theorem 3.1. Let $K$ be a nonempty, compact and convex subset of $X$ and let the mapping

$f: L(X, Y) \times K \times K \rightarrow Y$ be a $C$-convex function in the second argument. Assume that, for every $y \in K$, the set $\{x \in K: f(s, y, x) \in-C \backslash\{0\}, \forall s \in T x\}$ is open in $K$. Then, $\mathrm{GS}(1)$ has a solution.

Proof. We proceed by contradiction. Assume that GS(1) admits no solution, then for each $x_{0} \in K$, there exists some $y \in K$ and for all $s \in T x_{0}$ such that

$$
f\left(s, y, x_{0}\right) \in-C \backslash\{0\} .
$$

For every $y \in K$, define the set $N_{y}$ as follows:

$$
N_{y}=\{x \in K: f(s, y, x) \in-C \backslash\{0\}, \forall s \in T(x)\} .
$$

By given assumption, the set $N_{y}$ is open in $K$ and hence from (4), it follows that $\left\{N_{y}: y \in K\right\}$ is an open cover of $K$. Since $K$ is compact, there exists a finite set $\left\{y_{1}, y_{2}, \cdots, y_{n}\right\} \subset K$ such that $K=\bigcup_{i=1}^{n} N_{y_{i}}$. So there exists a continuous partition of unity $\left\{\beta_{1}, \beta_{2}, \cdots, \beta_{n}\right\}$ subordinate to $\left\{N_{y_{1}}, N_{y_{2}}, \cdots, N_{y_{n}}\right\}$ such that for all $x \in K$, $\beta_{j}(x) \geq 0(j=1,2, \cdots, n)$ with $\sum_{j=1}^{n} \beta_{j}(x)=1$ and $\beta_{j}(x)=0 \quad$ whenever $\quad x \notin N_{y_{j}}, \quad \beta_{j}(x)>0 \quad$ whenever $x \notin N_{y_{j}}$.

Define a mapping $h: K \rightarrow Y$ by

$$
h(x)=\sum_{j=1}^{n} \beta_{j}(x) y_{j}, \forall x \in K .
$$

Since $\beta_{j}$ is continuous for each $j$, it follows from (6) that $h$ is also continuous. Let $S:=\operatorname{Co}\left\{y_{1}, y_{2}, \cdots, y_{n}\right\} \subset K$. Then $S$ is a simplex of a finite dimensional space and $h$ maps $S$ into $S$. By Theorem 1.1, there exists some $x_{0} \in S$ such that $h\left(x_{0}\right)=x_{0}$.

Next define a mapping $g: L(X, Y) \times K \rightarrow Y$ by $g(s, x)=f(s, h(x), x)$. Since $f$ is a $C$-convex function in the second argument, by (6), we have

$$
g(s, x) \in \sum_{j=1}^{n} \beta_{j}(x) f\left(s, y_{j}, x\right)-C, \forall x \in K .
$$

For any given $x \in K$, let $K_{0}=\left\{j: x \in N_{y_{j}}\right\}$. Clearly, $K_{0} \neq \varnothing$. since $x_{0} \in S$ is a fixed point of $h$, it follows from (4) and (7) that for all $x \in K$,

$$
\begin{aligned}
0 & =f\left(s, x_{0}, x_{0}\right)=g\left(s, x_{0}\right)=f\left(s, h\left(x_{0}\right), x_{0}\right) \\
& =f\left(s, \sum_{j=1}^{n} \beta_{j}\left(x_{0}\right) y_{j}, x_{0}\right) \\
& \in \sum_{j=1}^{n} \beta_{j}\left(x_{0}\right) f\left(s, y_{j}, x_{0}\right)-C \subset-C \backslash\{0\}-C \\
& =-C \backslash\{0\} .
\end{aligned}
$$

That is, $0 \in-C \backslash\{0\}$, a contradiction. Hence, GS(1) has a solution. This completes the proof.

Example 3.1. Let $X=R, K=[0,1], Y=R^{2}, C=R_{+}^{2}$, 


$$
\begin{gathered}
\eta(y, x)=y-\sqrt{2} x, T(x)=\left(\begin{array}{cc}
x_{1} & 0 \\
0 & x_{2}
\end{array}\right), \forall x=\left(x_{1}, x_{2}\right) \in K . \\
f(s, y, x)=\langle T x, \eta(y, x)\rangle=\left(\begin{array}{cc}
x_{1} & 0 \\
0 & x_{2}
\end{array}\right)(y-\sqrt{2} x) \\
=\left(\begin{array}{c}
x_{1}(y-\sqrt{2} x) \\
x_{2}(y-\sqrt{2} x)
\end{array}\right), \forall x, y \in K .
\end{gathered}
$$

For all $\lambda \in[0,1]$ and $y_{1}, y_{2} \in K$,

$$
\begin{aligned}
& f\left(s, \lambda y_{1}+(1-\lambda) y_{2}, x\right)-\lambda f\left(s, y_{1}, x\right) \\
- & (1-\lambda) f\left(s, y_{2}, x\right) \\
= & \left(\begin{array}{c}
x_{1}\left(\lambda y_{1}+(1-\lambda) y_{2}-\sqrt{2} x\right) \\
x_{2}\left(\lambda y_{1}+(1-\lambda) y_{2}-\sqrt{2} x\right)
\end{array}\right)-\left(\begin{array}{l}
x_{1}\left(\lambda y_{1}-\sqrt{2} \lambda x\right) \\
x_{2}\left(\lambda y_{2}-\sqrt{2} \lambda x\right)
\end{array}\right) \\
& -\left(\begin{array}{c}
x_{1}\left((1-\lambda) y_{2}-(1-\lambda) \sqrt{2} x\right) \\
x_{2}\left((1-\lambda) y_{2}-(1-\lambda) \sqrt{2} x\right)
\end{array}\right) \\
= & 0 \in-C,
\end{aligned}
$$

i.e.,

$$
\begin{aligned}
& f\left(s, \lambda y_{1}+(1-\lambda) y_{2}, x\right) \\
& \in \lambda f\left(s, y_{1}, x\right)+(1-\lambda) f\left(s, y_{2}, x\right)-C .
\end{aligned}
$$

Then it is easy to see that $f$ is a $C$-convex function in the second argument, and the

$$
\begin{aligned}
& \{x \in K: f(s, y, x) \in-C \backslash\{0\}\} \\
& =\{x \in K: y-\sqrt{2} x<0\}=\left(\frac{\sqrt{2}}{2} y, 1\right]
\end{aligned}
$$

is open in $K$. Now we could say that all conditions of Theorem 2.1 hold. And also, the solutions set for GS(1) in Theorem 2.1 is $D=\{x \in K: y-\sqrt{2} x \geq 0\}=\left[0, \frac{\sqrt{2}}{2} y\right]$, $\forall y \in K$.

Theorem 3.2. Let $K$ be a nonempty, closed and convex subset of $X$ and let the mapping $f: L(X, Y) \times K \times K \rightarrow Y$ be a $C$-convex function in the second argument. Assume that:

1) For every $y \in K$, the set $\{x \in K: f(s, y, x) \in-C \backslash\{0\}, \forall s \in T x\}$ is open.

2) $K$ is locally compact and there is an $r>0$ and $x_{0} \in K,\left\|x_{0}\right\|<r$, such that, for all $y \in K,\|y\|=r$, there exists an $s \in T x$ such that $f\left(s, x_{0}, y\right) \in-C$.

Then, GS(1) has a solution.

Proof. Let $K_{r}:=\{x \in K:\|x\| \leq r\}$. Since $K$ is locally compact, $K_{r}$ is compact; hence, it follows from Theorem 2.1 that there exists an $\bar{x} \in K_{r}$, for all $y \in K_{r}$ there exists $s \in T \bar{x}$ such that

$$
f(s, y, \bar{x}) \notin-C \backslash\{0\} .
$$

We claim that $\bar{x}$ is the desired solution of $\mathrm{GS}(1)$. Indeed:

1) If $\|\bar{x}\|=r$, by the assumption 2), there exists $s \in T \bar{x}$ such that

$$
f\left(s, x_{0}, \bar{x}\right) \in-C .
$$

For any $y \in K$, choose $\lambda \in(0,1]$ such that $y_{\lambda}:=\lambda y+(1-\lambda) x_{0} \in K_{r}$. Then, from (7), it follows that $f\left(s, y_{\lambda}, \bar{x}\right) \notin-C \backslash\{0\}$. Since $f$ is a $C$-convex function in the second argument, we have

$$
\begin{aligned}
f\left(s, y_{\lambda}, \bar{x}\right) & \in \lambda f(s, y, \bar{x})+(1-\lambda) f\left(s, x_{0}, \bar{x}\right)-C \\
\lambda f(s, y, \bar{x}) & \in[Y \backslash(-C \backslash\{0\})]+(1-\lambda) C+C \\
& \subseteq Y \backslash(-C \backslash\{0\})+C=Y \backslash(-C \backslash\{0\}) .
\end{aligned}
$$

Implying that

$$
f(s, y, \bar{x}) \notin-C \backslash\{0\} .
$$

2) If $\|\bar{x}\|<r$, for any $y \in K$, choose $\lambda \in(0,1]$ such that $y_{\lambda}:=\lambda y+(1-\lambda) \bar{x} \in K_{r}$. Then, it follows from (7) that $f\left(s, y_{\lambda}, \bar{x}\right) \notin-C \backslash\{0\}$. Since $f$ is a $C$-convex function in the second argument, we have

$$
\begin{aligned}
f\left(s, y_{\lambda}, \bar{x}\right) & \in \lambda f(s, y, \bar{x})+(1-\lambda) f(s, \bar{x}, \bar{x})-C \\
& \subseteq \lambda f(s, y, \bar{x})-C \\
& \Rightarrow f(s, y, \bar{x}) \notin-C \backslash\{0\} .
\end{aligned}
$$

This completes the proof.

The following example shows that the assumption that the set $\{x \in K: f(s, y, x) \in-C \backslash\{0\}, \forall s \in T x\}$ is open in $K$, for every $y \in K$, is not trivial. For two similar examples for a vector-valued function, see [2,6].

Example 3.2. Let $X=R, K=[0,1], Y=R^{2}, C=R_{+}^{2}$, $\eta(y, x)=2 y-2 x, T(x)=\left(\begin{array}{cc}x_{1}^{2} & 0 \\ 0 & x_{2}^{2}\end{array}\right), \forall x=\left(x_{1}, x_{2}\right) \in K$,

$$
\begin{aligned}
f(s, y, x) & =\langle T x, \eta(y, x)\rangle=\left(\begin{array}{cc}
x_{1}^{2} & 0 \\
0 & x_{2}^{2}
\end{array}\right)(2 y-2 x) \\
& =\left(\begin{array}{c}
x_{1}^{2}(2 y-2 x) \\
x_{2}^{2}(2 y-2 x)
\end{array}\right), \forall x, y \in K,
\end{aligned}
$$

$$
\begin{aligned}
f(s, y, x)+f(s, x, y) & =\left(\begin{array}{l}
x_{1}^{2}(2 y-2 x) \\
x_{2}^{2}(2 y-2 x)
\end{array}\right)+\left(\begin{array}{l}
y_{1}^{2}(2 x-2 y) \\
y_{2}^{2}(2 x-2 y)
\end{array}\right) \\
& =\left(\begin{array}{l}
\left(x_{1}^{2}-y_{1}^{2}\right)(2 y-2 x) \\
\left(x_{2}^{2}-y_{2}^{2}\right)(2 y-2 x)
\end{array}\right) \leq 0 .
\end{aligned}
$$

$f$ is continuous and monotone in the sense that $f(s, y, x)+f(s, x, y) \leq 0$ for all $x, y \in K$. Also, it is 
easy to see that, for each $y \in K$, the set

$\{x \in K: f(s, y, x) \in-C \backslash\{0\}\}=(y, 1]$ and hence is open in $K$.

Next, we define following concepts which will be used in the sequel.

Definition 3.1. A mapping $f: L(X, Y) \times K \times K \rightarrow Y$ is said to be $C$-strong pseudomonotone respect to $T: K \rightarrow 2^{L(X, Y)}$ if, for any $x, y \in K$, there exists $u \in T x$, $f(u, y, x) \notin-C \backslash\{0\}$ implies $f(v, x, y) \in-C, \forall v \in T y$.

Remark 3.1. This definition generalizes corresponding definitions of $[9,10]$.

Example 3.3. Let $X=Y=R^{2}, K=R_{+}, C=R_{+}^{2}$, $f(s, y, x)=\langle T(x), \eta(y, x)\rangle, \eta(y, x)=y-\left(x+x^{3}\right)$,

$T(x)=\left(\begin{array}{cc}x_{1}+\frac{1}{x_{1}} & 0 \\ 0 & x_{2}+\frac{1}{x_{2}}\end{array}\right), \forall x=\left(x_{1}, x_{2}\right) \in K, u \in T x$.

$$
\begin{aligned}
f(u, y, x) & =\left(\begin{array}{cc}
x_{1}+\frac{1}{x_{1}} & 0 \\
0 & x_{2}+\frac{1}{x_{2}}
\end{array}\right)\left(y-\left(x+x^{3}\right)\right) \\
& =\left(\begin{array}{l}
\left(x_{1}+\frac{1}{x_{1}}\right)\left(y-\left(x+x^{3}\right)\right) \\
\left(x_{2}+\frac{1}{x_{2}}\right)\left(y-\left(x+x^{3}\right)\right)
\end{array}\right) \notin-C \backslash\{0\} .
\end{aligned}
$$

That implies that $y \geq x+x^{3}>x$, it follows that

$$
f(v, x, y)=\left(\begin{array}{l}
\left(y_{1}+\frac{1}{y_{1}}\right)\left(x-\left(y+y^{3}\right)\right) \\
\left(y_{2}+\frac{1}{y_{2}}\right)\left(x-\left(y+y^{3}\right)\right)
\end{array}\right) \in-C, \forall v \in T y .
$$

Hence, $f$ is $C$-strong pseudomonotone. For a similar example for a vector-valued function, see [6].

Definition 3.2. A mapping $f: K \rightarrow Y$, is said to be $-C$-continuous at $x \in K$ if, for each neighbourhood of nullelement in $Y$, there exists a neighbourhood of $X$, such that

$$
f(\tilde{x}) \in f(x)+V-C, \forall \tilde{x} \in U \cap K .
$$

If $f$ is $-C$-continuous at each point of $K$, then $f$ is $-C$-continuous on $K$.

Definition 3.3. Let $W, E$ be two topological spaces, a set-valued mapping $T: W \rightarrow 2^{E}$ is upper semicontinuous at $x \in W$ if, for each neighbourhood $V$ of $T x$, there exists a neighbourhood $U$ of $x$, such that $T(U) \in V$. If $T$ is upper semicontinuous at each point of $W$, then $T$ is upper semicontinuous on $W$.

Lemma 3.1 [11]. Let $W, E$ be two topological spaces, a set-valued mapping $T: W \rightarrow 2^{E}$ is compact valued, then $T$ is said to be upper semicontinuous at $x \in W$ if and only if: for any net $\left\{x_{\alpha}\right\} \subset W, x_{\alpha} \rightarrow x$, and $\left\{y_{\alpha}\right\}$, $y_{\alpha} \in T\left(x_{\alpha}\right)$, there exists a setnet $\left\{y_{\beta}\right\}$ of $\left\{y_{\alpha}\right\}$, such that $y_{\beta} \rightarrow y$.

Now we prove the existence result for GS(2) with monotonicity. First, we prove the following Minty's type lemma for GS(2).

Lemma 3.2. Let $K$ be a nonempty closed and convex subset of $X, Y$ be a real Banach space ordered by a nonempty closed convex pointed cone $C$ with apex at the origin and int $C \neq \phi$. Let $f: L(X, Y) \times K \times K \rightarrow Y$ be a trifunction, $T: K \rightarrow 2^{L(X, Y)}$ be a nonempty compact set-valued mapping. Suppose the following conditions hold:

1) $f(u, x, x) \in C, \forall u \in T x, x \in K$;

2) $f(u, y, x)$ is $C$-convex function in the second argument;

3) $T$ is upper semicontinous;

4) $f(u, y, x)$ is $-C$-continuous with respect to $(u, x)$, $\forall u \in T x, x, y \in K$.

Then there exists $x \in K$ and $u \in T x$, such that

$$
f(u, y, x) \notin-C \backslash\{0\}, \forall y \in K .
$$

if and only if there exists $x \in K$ such that

$$
f(v, x, y) \in-C, \forall y \in K, v \in T y .
$$

Proof. (9) $\Rightarrow(10)$ : It follows from the $C$-strong pseudomonotonicity of $f$ respect to $T$.

$(10) \Rightarrow(9)$ : Suppose that there exists $x_{0} \in K$ such that

$$
f\left(v, x_{0}, y\right) \in-C, \forall y \in K, v \in T y .
$$

If $x_{0} \in K$ is not the solution of (9), that is to say, $\exists \bar{y} \in K, \forall u \in T x$, we have

$$
f\left(u, y, x_{0}\right) \in-C \backslash\{0\} .
$$

For any given $y \in K$, we set $y_{t}=t \bar{y}+(1-t) x_{0} \in K$, $\forall t \in(0,1)$, for each $v_{t} \in T y_{t}$, it follows that

$$
f\left(v_{t}, x_{0}, y_{t}\right) \in-C \text {. }
$$

Since $f$ is $C$-convex function in the second argument, we have

$$
f\left(v_{t}, y_{t}, y_{t}\right) \in t f\left(v_{t}, \bar{y}, y_{t}\right)+(1-t) f\left(v_{t}, x_{0}, y_{t}\right)-C \text {. }
$$

It follows from inclusions (13), (14) and condition 1), we obtain

$$
\begin{aligned}
t f\left(v_{t}, \bar{y}, y_{t}\right) & \in f\left(v_{t}, y_{t}, y_{t}\right)-(1-t) f\left(v_{t}, x_{0}, y_{t}\right)+C \\
& \in C+C+C=C .
\end{aligned}
$$

Implying that

$$
f\left(v_{t}, \bar{y}, y_{t}\right) \in C, \forall t \in(0,1) .
$$

From $T$ is upper semicontinous and $y_{t} \rightarrow x_{0}, \forall v_{t} \in T y_{t}$, 
as $t \rightarrow 0^{+}$, we obtain that $\left\{v_{t}\right\}$ has a setnet (for simplicity, still denoted $\left.\left\{v_{t}\right\}\right)$ and there exists $u_{0} \in T x$, such that $v_{t} \rightarrow u_{0}$.

If we could prove

$$
f\left(u_{0}, \bar{y}, x_{0}\right) \in C \text {. }
$$

we will obtain a contradiction between (16) and (12), as $C$ is convex pointed cone. That will complete the proof.

In fact, suppose on the contrary that

$$
f\left(u_{0}, \bar{y}, x_{0}\right) \notin C \text {. }
$$

Since $C$ is closed, we know that there exists a origin neighbourhood $V$ in $Y$, such that

$$
\left[f\left(u_{0}, \bar{y}, x_{0}\right)+V\right] \cap C=\phi .
$$

Since $C$ is convex cone, it follows that

$$
\left[f\left(u_{0}, \bar{y}, x_{0}\right)+V-C\right] \cap C=\phi .
$$

From 4), there exists neighbourhood $U\left(u_{0}\right) \times U\left(x_{0}\right)$ of $\left(u_{0}, x_{0}\right)$ such that

$$
\begin{aligned}
& f(\bar{u}, \bar{y}, \bar{x}) \in f\left(u_{0}, \bar{y}, x_{0}\right)+V-C, \\
& \forall(\bar{u}, \bar{x}) \in U\left(u_{0}\right) \times U\left(x_{0}\right) .
\end{aligned}
$$

Since $v_{t} \rightarrow u_{0}, \quad y_{t} \rightarrow x_{0}$, as $t \rightarrow 0^{+}, \quad \exists \delta \in(0,1)$, such that

$$
\left(v_{t}, y_{t}\right) \in U\left(u_{0}\right) \times U\left(x_{0}\right), \text { as } 0<t<\delta .
$$

Thus from (18) we get

$$
f\left(v_{t}, \bar{y}, y_{t}\right) \in f\left(u_{0}, \bar{y}, x_{0}\right)+V-C .
$$

it follows (17) and (19) that

$$
f\left(v_{t}, \bar{y}, y_{t}\right) \notin C .
$$

which leads a contradiction to (15). This completes the proof.

Theorem 3.3. Let $K$ be a nonempty, closed, bounded and convex subset of a real reflexive Banach space $X$ and let the mapping $f: L(X, Y) \times K \times K \rightarrow Y$ be a $C$-convex function in the second argument and $C$-strong pseudomonotone. $T: K \rightarrow 2^{L(X, Y)}$ be a nonempty compact setvalued mapping. Then, GS(2) has a solution. by

Proof. Define two set-valued mappings, $A, B: K \rightarrow 2^{K}$

$$
\begin{aligned}
A(y)= & \{x \in K: f(u, y, x) \notin-C \backslash\{0\}, \\
& \text { for some } u \in T x\}, \forall y \in K .
\end{aligned}
$$

and

$$
\begin{aligned}
& B(y)=\{x \in K: f(v, x, y) \in-C, \text { for all } v \in T y\}, \\
& \forall y \in K .
\end{aligned}
$$

Clearly, $A(y)$ and $B(y)$ both are nonempty since $y \in A(y) \cap B(y)$ for all $y \in K$.
We claim that $A$ is KKM-mapping. If assertion were false, then there would exists $\left\{x_{1}, x_{2}, \cdots, x_{n}\right\} \subset K$ and $\lambda_{i} \geq 0,1 \leq i \leq n$, with $\sum_{i=1}^{n} \lambda_{i}=1$ such that $x=\sum_{i=1}^{n} \lambda_{i} x_{i} \notin \bigcup_{i=1}^{n} A\left(x_{i}\right)$. Hence for any $u \in T x$, we have $x \notin A\left(x_{i}\right)$, for each $i$.

Implying that, $f\left(u, x_{i}, x\right) \in-C \backslash\{0\} \quad \forall i$. Thus, $\sum_{i=1}^{n} \lambda_{i} f\left(u, x_{i}, x\right) \in-C \backslash\{0\}$. Since $f$ is a $C$-convex function in the second argument, we have

$$
\begin{aligned}
0 & =f(u, x, x)=f\left(u, \sum_{i=1}^{n} \lambda_{i} x_{i}, x\right) \\
& \in \sum_{i=1}^{n} \lambda_{i} f\left(u, x_{i}, x\right)-C \subseteq-C \backslash\{0\}-C=-C \backslash\{0\} .
\end{aligned}
$$

which is a contradiction. Hence $A$ is a KKM-mapping.

Since $f$ is $C$-strong pseudomonotone, it follows that $A(y) \subset B(y)$ for all $y \in K$, and hence $B$ is also a KKM-mapping. By Lemma 2.1, we see that

$$
\bigcap_{y \in K} A(y)=\bigcap_{y \in K} B(y) .
$$

Next we claim that for each fixed $y \in K, B(y)$ is bounded, convex and closed in $K$. Indeed, $B(y)$ is bounded as $B(y) \subset K$. Let $x_{1}, x_{2} \in B(y)$, then we have

$$
f\left(v, x_{1}, y\right) \in-C \text { and } f\left(v, x_{2}, y\right) \in-C
$$

Since $f$ is $C$-convex function in second argument, we have that, for $\lambda \in(0,1]$,

$$
\begin{aligned}
& f\left(v, \lambda x_{1}+(1-\lambda) x_{2}, y\right) \\
& \in \lambda f\left(v, x_{1}, y\right)+(1-\lambda) f\left(v, x_{2}, y\right)-C \subseteq-C .
\end{aligned}
$$

This implies that $B(y)$ is convex for each fixed $y \in K$. The continuity of $f$ in the second argument and closedness of $-C$ give the closedness of $B(y)$. We now equip $X$ with that weak topology. Since $B(y)$ is closed, bounded and convex subset of the reflexive Banach space $X$, then it turns out to be weakly compact for all $y \in K$. Hence by Theorem 1.2, we have $\bigcap_{y \in K} B(y) \neq \phi$. This implies that there exists $x_{0} \in K$, such that

$$
f(v, x, y) \in-C, \forall y \in K, v \in T y .
$$

Therefore by Minty's type Lemma 3.2, we conclude that there exists $x_{0} \in K$ and $u_{0} \in T x_{0}$ such that

$$
f\left(u_{0}, y, x\right) \notin-C \backslash\{0\}, \forall y \in K .
$$

That is to say GS(2) has a solution. This completes the proof.

Remark 3.2. Let $f(s, y, x)=\langle T x, \eta(y, x)\rangle, \forall x, y \in K$, where $T: K \rightarrow L(X, Y)$ is a single-valued mapping, $\eta: K \times K \rightarrow X$ is a nonlinear mapping, then GS(1) and $\mathrm{GS}(2)$ both reduces to the SVVLIP(3). As applications, we have the following existence result for $\operatorname{SVVLIP(3).~}$ 
Corollary 3.1. Let $K, X, T, \eta$ be as in Theorem 3.1 and Remark 3.2, let $\eta$ be affine in the first argument. For each $y \in K$, the set $\{x \in K:\langle T x, \eta(y, x)\rangle \in-C \backslash\{0\}\}$ is open in $K$. Then the $\operatorname{SVVLIP(3)}$ has a solution.

Corollary 3.2. Let $K, X, T, \eta$ be as in Corollary 3.1. Assume that:

1) For every $y \in K$, the set

$\{x \in K:\langle T x, \eta(y, x)\rangle \in-C \backslash\{0\}\}$ is open.

2) $K$ is locally compact and there is an $r>0$ and $x_{0} \in K,\left\|x_{0}\right\|<r$, such that for all $y \in K,\|y\|=r$, $\left\langle T y, \eta\left(x_{0}, y\right)\right\rangle \in-C$.

Then, the SVVLIP(3) has a solution.

\section{REFERENCES}

[1] F. Giannessi, "Theorem of Alternative, Quadratic Programs, and Complementarity Problems," In: R. W. Cottle, F. Giannessi and J. L. Lions, Eds., Variational Inequality and Complementarity Problems, John Wiley and Sons, Chichester, 1980, pp. 151-186.

[2] Y.-P. Fang and N.-J. Huang, "Strong Vector Variational Inequalities in Banach Spaces," Applied Mathematics Letters, Vol. 19, No. 4, 2006, pp. 362-368. doi:10.1016/j.aml.2005.06.008

[3] X. J. Long, N. J. Huang and K. L. Teo, "Existence and Stability of Solutions for Generalized Strong Vector
Quasi-Equilibrium Problem," Mathematical and Computer Modelling, Vol. 47, No. 3-4, 2008, pp. 445-451. doi:10.1016/j.mcm.2007.04.013

[4] J. Li and Z. Q. He, "Gap Functions and Existence of Solutions to Generalized Vector Variational Inequalities," Applied Mathematics Letters, Vol. 18, 2005, pp. 9891000. doi:10.1016/j.aml.2004.06.029

[5] E. Blum and W. Oettli, "From Optimization and Variational Inequalities to Equilibrium Problems," Math Students, Vol. 63, No. 1-4, 1994, pp. 123-145.

[6] K. R. Kazmi and S. A. Khan, "Existence of Solutions to a Generalized System," Journal of Optimization Theory and Applications, Vol. 142, No. 2, 2009, pp. 355-361. doi:10.1007/s10957-009-9530-7

[7] K. Fan, "A Generalization of Tychonoff's Fixed-Point Theorem," Mathematische Annalen, Vol. 142, 1961, pp. 305-310. doi:10.1007/BF01353421

[8] L. E. J. Brouwer, "Zur Invarianz des $n$-Dimensional Gebietes," Mathematische Annalen, Vol. 72, 1912, pp. 55-56. doi:10.1007/BF01456889

[9] Q. G. Liao and J. Y. Fu, "Systems of Generalized Vector Quasi-Equilibrium Problems with Pseudo-Monotone Mappings," Journal of Nanchang University (Natural Science), Vol. 35, No. 1, 2011, pp. 12-15.

[10] F. Giannessi, "Vector Variational Inequalities and Vector Equilibria," Mathematical Theoreies, Kluwer Academic Publishers, Berlin, 2000. 